\title{
A WWW based Certification Infrastructure for Secure Open Network Transactions
}

\author{
Tomas Gustavsson
}

Department of Computer and System Sciences

Stockholm University \& Royal Institute of Technology

Electrum 230, 16440 Kista, Sweden

Phone : + 468161701

Fax : + 4687039025

E-mail :tomasg@dsv.su.se

\begin{abstract}
This paper describes the development of a WWW based interface to a Public Key Infrastructure. The WWW interface can be used to provide easy user access to certification functions, as well as automatic on-line functionality for various security enhanced applications. The paper describes the certification system and its use based on the WWW interface.
\end{abstract}

Keywords

Public Key Infrastructure, WWW, X.509, PEM, CMS

\section{INTRODUCTION}

Public Key Infrastructures (PKI) has received lately a lot of attention and projects to build publicly available infrastructures are being started. Certificates, defined in the X.509 standard, have been adopted as the form for transportation of public keys. Therefore certificates are becoming used in all kinds of security protocols and applications, and protocols and tools for their handling are being developed. The process of handling certificates and making them available to security enhanced applications requires a range of certification functions and 
protocols. Different applications use different mediums for transporting data, and therefore the certification infrastructure must also support a variety of transport protocols. An application that has grown to be the most popular application on the Internet is the World Wide Web. Security enhancements for the WWW have become one of the largest potential users of the certification infrastructure.

\section{CERTIFICATION INFRASTRUCTURE}

\subsection{Introduction}

Many security protocols, in particular those that support widely distributed security services, require authenticated public keys. The public keys can be created, stored and distributed in the form of certificates. Administration of certificates includes their creation, storage, distribution, and verification.

The CCITT recommendations X.500-X.521 (1988) specify that creation of certificates should be performed by Certification Authorities (CAs). The format of certificates and the method for their verification are defined in the X.509 standard. The responsibility for storage and distribution of certificates is performed by X.500 directories, though as yet, such directories are not widely established.

RFC 1422 (Kent, 1993) supplements the X.509 standard with the protocol for creation and verification of certificates. It specifies an Internet wide hierarchical infrastructure of CAs. RFCs 1421-1424, Linn (1993), Kent (1993), Balenson (1993), Kaliski (1993), define a set of functions for the administration of certificates. They define the structure of PEM letters, which are themselves a medium for the exchange of certificates. The PEM description of the administrative functions does not address the problem of certificate storage in the absence of the X.500 directories.

\subsection{The CMS structure}

A Certificate Management System (CMS) is comprised of a number of co-operating CAs. The CA's principle role is in signing certificates, either of users or of other CAs, and thereby testifying that the certificate has a legitimate binding to the owner's Distinguished Name (DN) (CCITT, 1988). In RFC 1422 CAs are organized in a hierarchical infrastructure. This simplifies certificate verification, since all certificate verification paths within the system are known to converge, in the worst case at the top of the hierarchy.

The certification infrastructure whose implementation is described in this paper is a strict hierarchy with arbitrary depth, where each CA is certified by only one parent CA.

\subsection{The roles of CMS agents}

Within the certification hierarchy, some CAs are given special roles and responsibilities (see Figure 1). The Policy Certification Authority (PCA) is the root of the hierarchy, which makes it the common point of trust for verification of all certificates in the system. The lowest level 
CAs are responsible for the administration of users. Because of their special role in relation to the users, these CAs are named User Certification Authorities (UCAs).

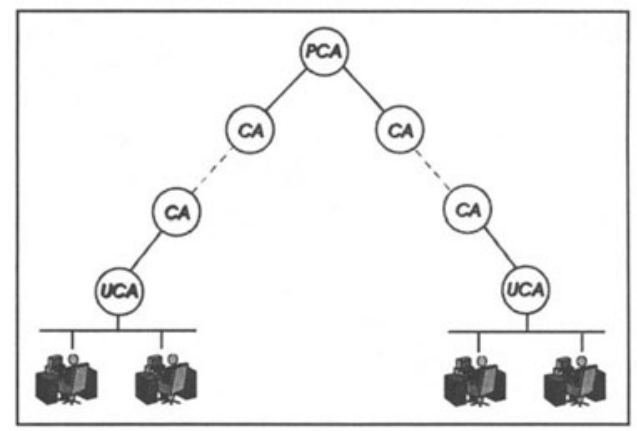

\section{Figure 1 A Certificate Management System.}

The PCA defines the security policy that all the CAs in its hierarchy are bound to follow. The policy specifies, among other things, technical and procedural security measures that are imposed on all the CAs and CMS agents of that hierarchy. In a global system, the PCA must be responsible for making its policy available to all users of the system. The importance of this role is reduced in autonomous hierarchies.

The PCA is responsible for the administration of the hierarchy structure. For each CA in the system, the PCA stores its DN and address*. The PCA uses this information to resolve certificate requests when the address of the certificate owner is not known.

A separate role of the PCA is to serve as a distribution point for Certificate Revocation Lists (CRLs) (Kent, 1993) of all CAs in its subhierarchy. Each CA and UCA periodically issues a CRL and sends it to the PCA. The users of the system can retrieve the CRLs from the PCA when needed for the verification of certificates.

The registration of users' DNs is performed by the UCAs. Other CAs are not allowed to administer users, which is more restrictive than the model described in the PEM specifications. Users register their DNs with their UCA. The UCA can autonomously ensure that the users DNs are unique, since the system imposes name subordination. The UCAs store the DNs and addresses of all the users they register.

The UCAs are the most suitable agents to provide for the expeditious storage and retrieval of user certificates in the absence of X.500 Directories. After signing user certificates, UCAs return them to the owners and store a copy locally. UCAs handle the distribution of user certificates, since they can automatically and immediately respond to requests for certificates. This service is based on the assumption that the UCAs' addresses are known to remote users or that they can somehow be derived or obtained.

\footnotetext{
* The form of the address depends on the means of communication used by the system. Our current implementation uses Internet e-mail addresses.
} 
Other CAs within the hierarchy do not perform any special functions. Their purpose is mainly to reflect the structure of the organization that runs the CMS sub-hierarchy (whether the organization is political, geographical or commercial). These CAs are not strictly necessary, as the PCA can certify UCAs directly. However, it is unlikely that such a flat structure would be preferred to a more distributed hierarchy of authorities.

The CMS User Agents (UAs) implement the interface between security applications and the CMS. They provide security applications with verified certificates either by retrieving them through the CMS or by reading them from a local certificate cache.

All CMS agents perform local caching of certificates. Each CA keeps copies of all the certificates in its certification path, as well as the certificates of all its immediate subordinates, i.e. those it has issued. Each CMS UA keeps copies of all the certificates that it has verified during its operative lifetime, for as long as they are valid.

\section{USING WWW WITH THE CERTIFICATION INFRASTRUCTURE}

\subsection{Introduction}

The current Internet standard protocol defined in RFC1422 is primarily based on e-mail for transportation of certification messages. Internet e-mail however is not very suitable for interactive procedures. This is due to the of-line nature of mailbox handling and the often substantial delays present in the e-mail system. The WWW system however is and interactive on-line protocol that today is responsible for most of the traffic on the Internet. Therefore is it natural to enhance the certification infrastructure with WWW based functionality. The system described here is an enhancement of the e-mail based system described by Kapidzic and Davidson (1995).

\subsection{Displaying the Infrastructure using WWW}

The certification infrastructure, due to its hierarchical structure, is very suitable for displaying and handling via the WWW system. With a WWW browser, it is easy to maneuver through the hierarchy searching for information or performing certification functions based on the HTTP protocol. As described earlier, CAs in the infrastructure can be divided into three categories:

- Top level PCA

- Intermediate level CAs

- The lowest level (user) CAs

There are some differences in the appearance of various CAs on the WWW from the users point of view. For the top level PCAs, which are functioning as CRL distribution points, there is the possibility to fetch CRLs. This function can not be performed with other CAs than the top level PCA, because all lower level CAs send their CRLs to the PCA for storage. One interesting function for users of the infrastructure is to be able to verify and fetch the true public key of the top of the certification path. The key can be displayed on a WWW page, but 
the distribution to CMS clients should be through out-of-band methods. Figure 2 shows the top level certification page of the company COST, Computer Security Technologies.

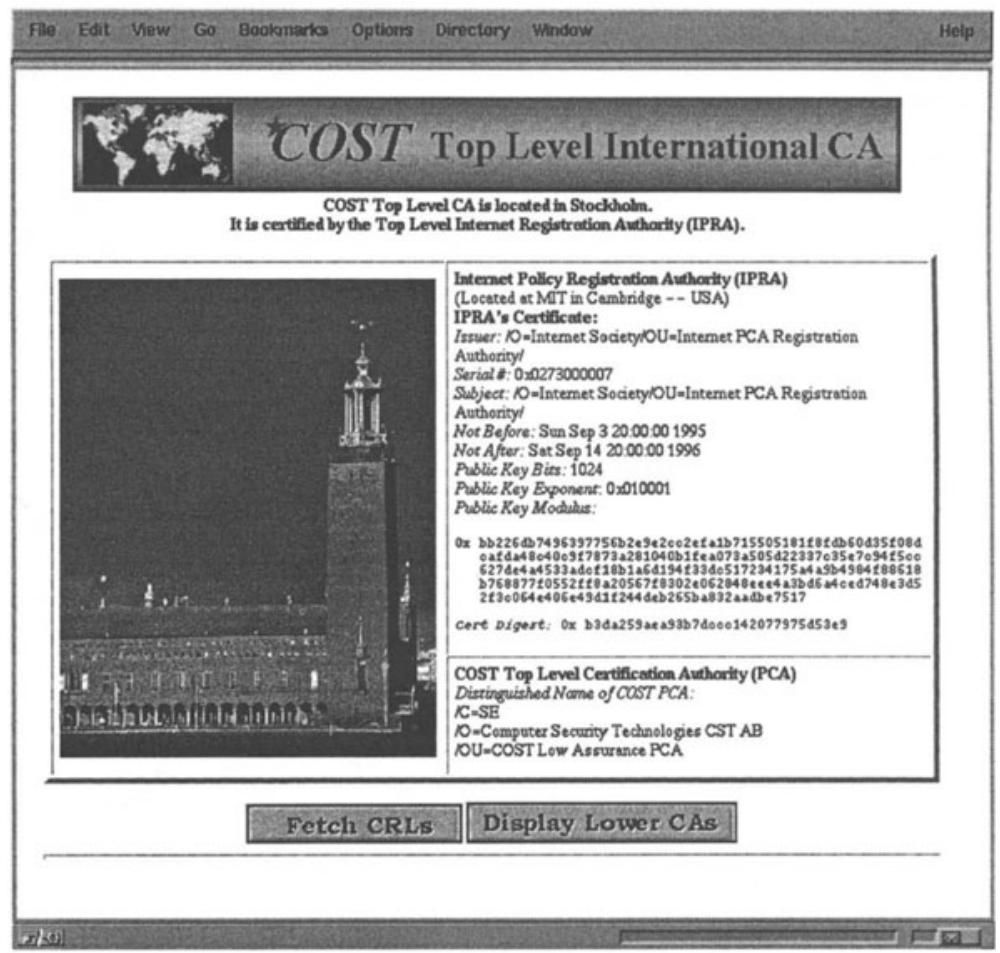

Figure 2 COST PCA Home page.

In the case when there exist lower level CAs in the infrastructure, these should be possible to view. Since all CAs of the certification hierarchy are registered with the PCA, the PCA can display information about all the subordinate CAs in its infrastructure and give the possibility to a user to move down to lower level CAs. Since the CAs at the level below the PCA are signed by the PCA, it could also be possible to fetch the certificates of those CAs. This however, will not be necessary if the lowest level CAs return the complete certification path when returning user certificates.

The intermediate level CAs don't have any special functions except to be a part of the certification path. Therefore their display consists only of general information about their registration entries and certificates, as well as the possibility to move on to CAs which are on levels even further below. The intermediate level CAs role is in this case strictly organizational, meaning that they exist only to uphold the organization of a large infrastructure. One useful 
feature, that could be easily obtained with an HTML anchor, could be a function to fetch the CRL of the current CA from the PCA. This however is also unnecessary when the PCA can display the lower CAs in a list for fetching CRLs, as we will see later. On the basis of their limited functionality, the intermediate level CAs can be bypassed by users browsing through the hierarchy, since they don't provide any useful user functions.

As mentioned earlier, the lowest level UCAs certify users. UCAs can display all users certified by them and provide the possibility to fetch their respective certificates. When a certificate is fetched the complete certification path is delivered, removing the need to fetch certificates from other CAs than the top of the certification path. Some other possible functions at these points in the infrastructure will be discussed in subsequent sections.

\subsection{Certification functions in the WWW}

In the global certification infrastructure some certification functions must be performed dynamically. The first use of the certification infrastructure is static, meaning that once a certificate has been issued, it is valid for as long as its validity period states. Also there are no possibilities for dynamically adding or revoking users certificates or for verification of the validity. In the global certification infrastructure all these function should be available. The system can also support dynamic revocation of certificates at users request, and also verification mechanisms in the same way.

Except for the obvious functions of fetching CRLs and certificates, other functions can be added to enhance the functionality of the WWW interface to the CMS. User functions that can be provided are the ability to certify users and to search the infrastructure for other parties. Certifying users means the creation of a users private and public key pair and creating a selfsigned certificate. Once the certificate has been prepared correctly, it is added to the infrastructure with the possibility to be used instantly by other parties in the global communication environment. Searching for parties in the infrastructure means automatically searching the infrastructure for certificates and registration entries. Based on this reasoning, there are four classes of functions that should be available to users of the infrastructure. Users of the infrastructure can be treated as both users or servers in this context.

1. Certifying users

2. Searching the infrastructure

3. Fetching certificates

4. Verifying certificates

Basic protocols for handling these certification functions have been and are being specified. Our development has so far been concentrated around the principles described in the RFC1422. In the original specification, the protocol for transporting certificate management messages is e-mail, but it can in most cases be extended by a system based on the HTTP protocol used in the WWW systems. 


\section{Certification Management Messages}

We have adopted the PEM letter format for transporting the certification management messages over the HTTP protocol. The PEM letter format is intended to be used over insecure channels and is therefore suitable for usage with the WWW protocols. The PEM letter format consists of a message body between delimiters, as shown in figure 4 .

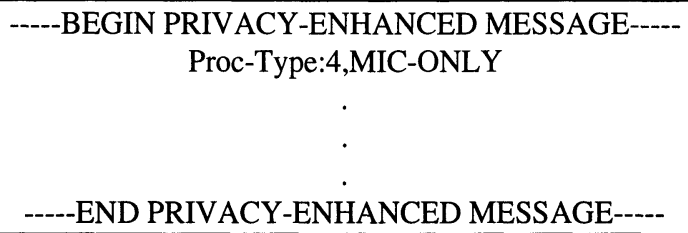

\section{Figure 4 PEM letter format.}

To process the received certification messages at the client side, we introduced a new HTTP MIME-type message, application/x-cms. When a message with this MIME type is received by the WWW client, it is configured to take appropriate action, e.g., launch an application capable of receiving the PEM formatted certification message and process it (Kapidzic, 1995). If extra non-security sensitive information is needed for the processing of the PEM message, it can be sent as headers before the PEM message delimiter. The creation of the PEM messages at the server side is implemented through CGI programs for the HTTP server. The CGI program can be invoked from references in HTML pages with different parameters for different certificate functions. The CGI program can also be used to create HTML pages for attractive presentation of the infrastructure.

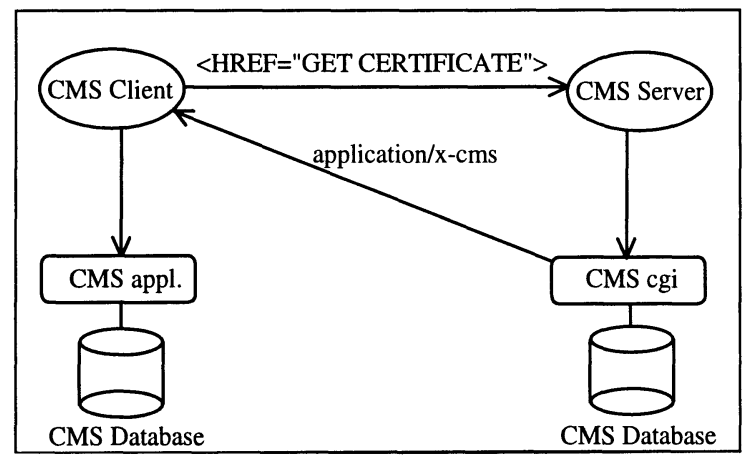

Figure 5 Requesting, receiving and verifying the certificates. 


\section{Fetching certificates}

When a user wants to communicate securely with another user in an open network, the user must obtain the partner's certificate. It can be obtained either automatically upon initial contact with the communicating partner or it can be fetched beforehand from a partner's UCA. When using on-line media, such as the WWW, the certificates can easily be moved automatically, but in case of off-line communication, such as e-mail, it would be an advantage to be able to quickly fetch certificates before contacting the other party. This is also the case when a user wants to encrypt the first message sent to the other party. In cases like these, the WWW is an excellent interface for interacting with the certification infrastructure.

Since fetching of certificates is done from the UCA an appropriate CGI program must be able to create a list of registered users whose certificates can be retrieved. This list is shown in figure 6

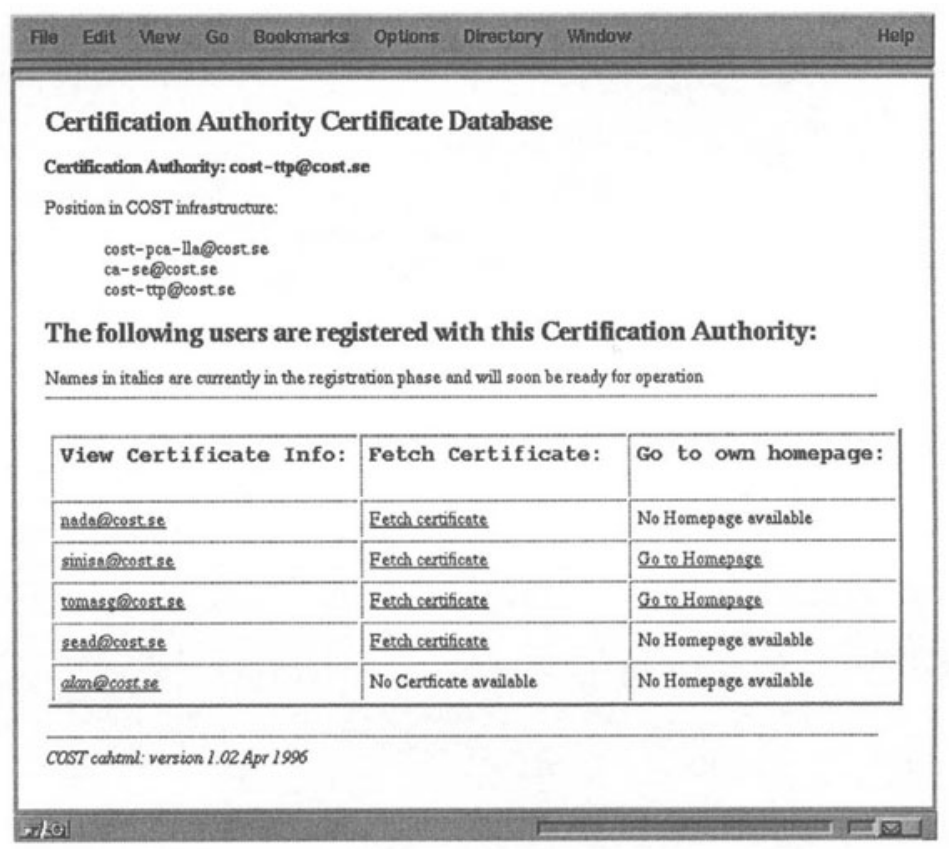

Figure 6 The list of users registered by the UCA.

The list consists of anchors to the CA server with parameters to display the certificate of a specified user. Clicking on a particular user causes a more detailed description of the user to appear. The detailed description contains the complete $\mathrm{DN}$ of the user, together with an option to fetch the actual certificate of the selected user. This is shown in figure 7. 


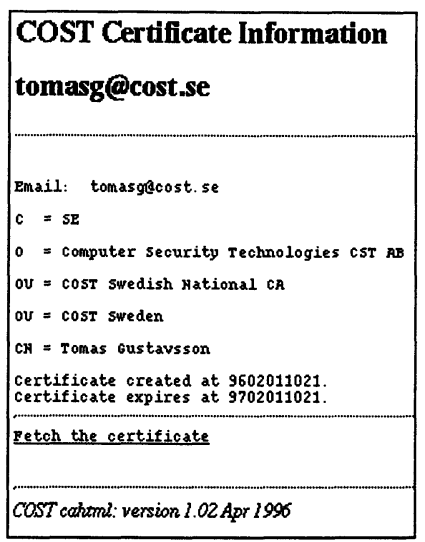

Figure 7 Detailed user description.

By choosing to fetch the certificate, a certificate management message with MIME type application $/ \mathrm{x}$-cms is sent to the WWW client for processing. When the certificate has been successfully verified, the user can continue with his/her intended communications.

\section{Verifying certificates}

In the RFC1422 trust model, certificate verification is based on usage of CRLs that are issued at regular time intervals. If a certificate was revoked after a CRL was issued and before a new one has been issued, the certificate may still be regarded as valid. With the WWW or some other on-line media, it could be possible to verify certificates towards the certification authority on-line. On-line certification introduces new problems and protocols which are currently being investigated, therefore in this paper we concentrate on the usage of traditional CRLs.

With a short CRL issuing time, decent credibility of certificates can be obtained. All CAs in the infrastructure are supposed to issue CRLs periodically, and when verifying a certificate, all CRLs in the certification path must be valid for the certificate to verify correctly. The display of the current COST certification hierarchy providing the possibility to fetch CRLs of CAs is shown in figure 8. 


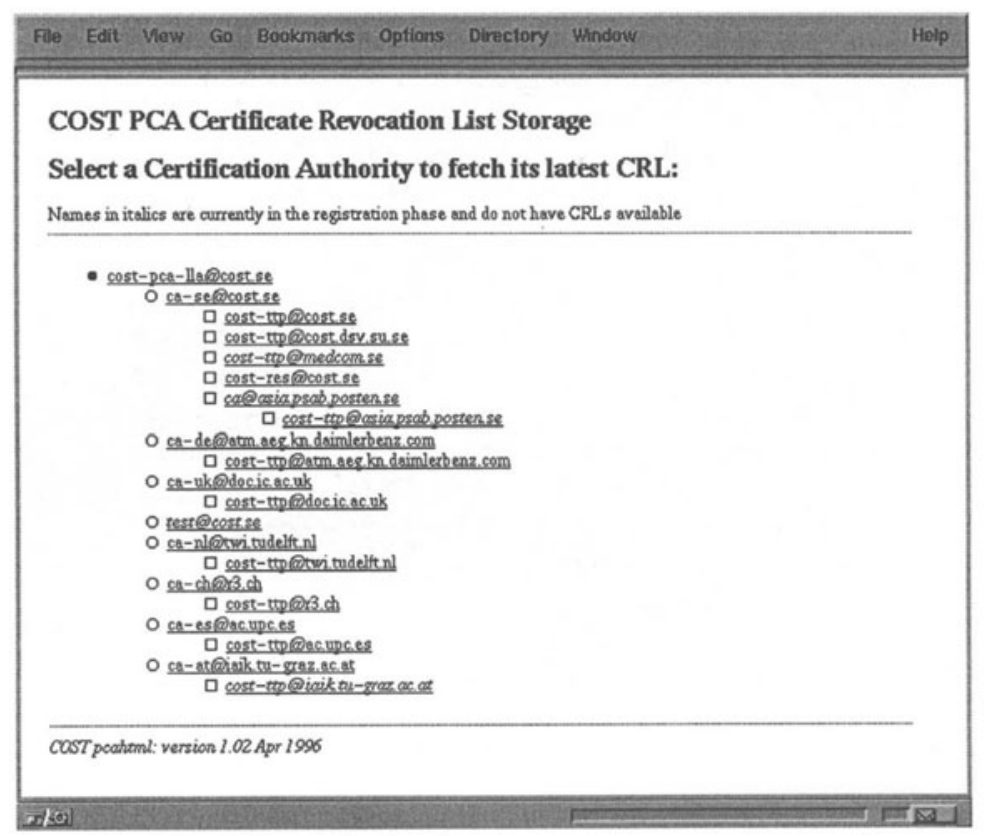

Figure 8 COST PCA display for fetching CRLs.

Similar to fetching of certificates, when a user requests a CRL of a specific CA by clicking at the PCAs WWW interface, the last issued CRL from that CA is sent to the user as a CMS message with MIME-type application/x-cms. Since the need for CRLs arise when the software is trying to verify a certificate, it is much more convenient if the software can fetch the CRLs automatically when needed. With the mechanism described here, this can be accomplished simply by issuing the correct HTTP request to the PCA.

\section{Certifying Users}

The procedure of certifying users in the infrastructure is a process with several steps. First, a secret/public key pair is generated, a certificate is created and sent to the UCA for signing. After receiving back the signed certificate the user must apprehend the CRLs for his own certification path. This procedure could be handled completely transparently for the user. With the traditional mail interface to the certification infrastructure, this procedure requires one letter to be sent from the user and at least two to be received (depending on the depth of the hierarchy). Receiving of the e-mail messages can be handled automatically, but the delay for the messages may be noticeable. Using the WWW as an interface to these functions can make the procedure both simple and instant. The same method as used by HTML forms can be used for submitting the Certificate Signature Request to the server. The server then sends back the 
Certificate Reply, witch is processed at the client side, instantly. Automatic fetching of the CRLs can be obtained as described earlier. One problem with using the WWW as an interface to this function is that it needs local processing to generate the user's key pair, something the HTML is not designed for. This currently creates certain difficulties for simple and transparent implementation of that function but with the upcoming of new enhancements to the WWW protocols, such as Java, this could be handled conveniently.

\section{Searching the infrastructure}

One benefit of the WWW system, except the possibility for automatic functions, are the interactive features provided to users of the infrastructure. Using the WWW is very suitable both for manual and automatic searching for communication partners. Manual searches are done by browsing the infrastructure and at the end finding the requested certificate. Automatic searches can be done effectively as demonstrated by various search engines deployed in the Internet. These kind of searches benefit from the limited structure of the certification infrastructure. The target results of a search through the infrastructure could be:

- A certificate

- A CRL (or similar verification functionality)

- Information about the other party, ex. e-mail address, URL, etc.

The information returned by the searches could be displayed to the user, or even processed automatically. These kinds of functions would benefit the users of a large certification infrastructure.

\section{IMPLEMENTATION ISSUES}

If the components of the certification infrastructure have already been implemented as e-mail based CA agents, providing access from the WWW for the most common function is easily done, if the same message form is used (as with PEM letter format). So far, in our implementation only fetching of certificates and CRLs has been implemented. This is because other described functions require extra implementation, outside of the basic certification functions.

A practical problem with on-line CA operations is handling of the CAs private key. If the private key needs to be available for the purpose of signatures, serious security concerns arise. The primary functions however does not require the use of the CAs private key. The functions for fetching certificates and CRLs can both be performed on-line without the need for additional CA signatures. The only function described here that cannot be performed without CAs private key is the on-line process of certifying users. 


\section{REFERENCES}

Balenson, D. (1993), "Privacy Enhancement for Internet Electronic Mail: Part 3: Algorithms, Modes, and Identifiers", $R F C$ 1423, TIS.

CCITT Recommendations X.500 - X.521 (1988), "Data Communication Networks Directory", November 1988

Kaliski, B. (1993), "Privacy Enhancement for Internet Electronic Mail: Part 4: Notary, Co-Issuer, CRL-Storing and CRL-Retrieving Services", RFC 1424, RSA Laboratories.

Kapidzic, N., Davidson, A. (1995), "A Certificate Management System: Structure, Functions and Protocols", Internet Society Symposium on Network and Distributed System Security.

Kapidzic, N. (1995), "Creating Security Applications Based on The Global Certificate Management System".

Kent, S. (1993), "Privacy Enhancement for Internet Electronic Mail: Part 2: Certificate Based Key Management", RFC 1422, BBN.

Linn, J. (1993), "Privacy Enhancement for Internet Electronic Mail: Part 1: Message Encryption and Authentication Procedures", RFC 1421., DEC

\section{BIOGRAPHY}

Tomas Gustavsson has a Master of Science degree in electrical engineering from the Royal Institute of Technology (KTH) in Stockholm. He has been working in the security laboratory of the Department of Computer- and System Sciences since 1994. He is also an employee of the company Computer Security Technologies CST AB (COST). 